\title{
Vitamin D Deficiency in Proximal Femur Fracture Patients of South-East Queensland
}

\begin{abstract}
Objectives: To determine the incidence of hypovitaminosis D in proximal femur fracture (PFF) patients and investigate if sociodemographic factors or radiographic parameters are associated with vitamin D levels.

Methods: This is a consecutive case series of South-East Queensland patients presenting with lowenergy PFFs. Vitamin D levels and sociodemographic factors (age, gender, postcode, medications, type of residence) were collected from medical records. Radiographic parameters included PFF type and cortical thickness of the femur.

Results: 313 patients were included (mean age $=79.5$ years) and $105(34 \%)$ were deficient in vitamin $\mathrm{D}(<50 \mathrm{nmol} / \mathrm{L})$. There was no association between vitamin D levels and sociodemographic factors or radiographic parameters. Eighty-four (84\%) of vitamin D deficient patients were not taking vitamin D supplements.
\end{abstract}

Conclusions: Social and demographic factors are not correlated with vitamin D levels in this cohort. Routine vitamin D supplementation may be indicated in ageing patients although it is not always protective of low-energy fractures.

Keywords: femoral fractures, hip fractures, Queensland, vitamin D, vitamin D deficiency 


\section{MAIN TEXT}

\section{Introduction:}

Vitamin D deficiency, defined as a serum 25-hydroxyvitamin D concentration of less than $50 \mathrm{nmol} / \mathrm{L}$, commonly occurs from a lack of exposure to sunlight. ${ }^{1}$ Vitamin D deficiency leads to reduced absorption of calcium from the gut which results in hypocalcemia and decreased bone mineralisation, a disease process known as osteomalacia. Additionally, parathyroid hormone levels rise in response to hypocalcemia and stimulates osteoclasts to increase bone resorption. As a result, vitamin D deficiency predisposes individuals to fractures in load-bearing areas of the skeleton such as the proximal femur. ${ }^{2}$

Proximal femur fractures (PFFs) represent a major burden to individuals, the community and the health system. Between July 2015 and June 2016, there were approximately 18,700 new hip fractures among Australians aged 45 years and above. ${ }^{3}$ Of these, $93 \%$ were from a falls-related injury and $87 \%$ were from low-energy falls. ${ }^{3}$ Low-energy trauma refers to falling from standing height or less, whereas high-energy trauma generally involves more forceful impacts or higher speeds such as motor vehicle accidents. ${ }^{4}$ This incidence is expected to rise due to an ageing population, underscoring the need for effective prevention strategies and pharmacological management of bone health.

The frequency of hypovitaminosis D amongst hip fracture patients has been well documented in other parts of the world such as Russia ${ }^{5}$, Switzerland ${ }^{6}$, Great Britain ${ }^{7-10}$, India ${ }^{11}$, Finland ${ }^{12}$ and Spain ${ }^{13}$ with reported rates of vitamin D deficiency ranging from $53 \%$ to $92 \%$. There is limited data in Australia with only one Tasmanian study from 2002 reporting that $67 \%$ of patients had vitamin D levels below $28 \mathrm{nmol} / \mathrm{L}^{14}$

The objectives of this study are to, firstly, compare the incidence of hypovitaminosis D in PFF patients from South-East Queensland with other reports globally. Secondly, to investigate if sociodemographic factors, femoral cortical thickness or fracture type are associated with vitamin D levels. Lastly, determine the rate and effectiveness of vitamin D supplementation in PFF patients.

\section{Methods:}

This is a retrospective analysis of a consecutive case series of patients with PFF that presented to a tertiary regional hospital in South-East Queensland between January 2018 to January 2019. Patients of any age that presented with a proximal femur fracture due to low-energy trauma and received a serum vitamin D level on admission were included. Patients with high-energy trauma and periprosthetic fractures were excluded. Each patient's serum 25-hydroxyvitamin D level was measured using liquid chromatographic separation followed by tandem mass spectrometry. The laboratory followed a series of internal and external quality control checks including standardisation through the Vitamin D External Quality Assessment Scheme (DEQAS). Age, gender, postcode, medications, type of residence and admission date were collected from medical records. Type of residence was 
classified as either nursing home (including assisted living) or private residence (including units in retirement villages). Admission date was used to detect seasonal variation in vitamin D levels of PFF patients. Patients were divided into three groups based on medications for bone health immediately before admission: calcium and vitamin D supplements only; antiresorptive medications (such as denosumab or bisphosphonates) with or without supplements; and no bone protection medications.

The Socio-Economic Indexes for Areas (SEIFA) is a report developed by the Australian Bureau of Statistics (ABS) that ranks areas in Australia according to their relative socioeconomic advantage or disadvantage ${ }^{15}$ Each postcode's rank is based on dimensions collected from Census data such as overall income, education, employment, occupation and housing. In this study, the Index of Relative Socio-Economic Advantage and Disadvantage within the SEIFA report was used to estimate the patients' level of socioeconomic status based on postcode recorded at admission.

Participants' radiographs were evaluated for combined cortical thickness (CCT) of the affected femur and fracture type. Femoral CCT was calculated by measuring the sum of the medial and lateral cortical thicknesses of the femur $3 \mathrm{~cm}$ below the lesser trochanter and dividing it by the total thickness of the shaft, to be consistent with previous reports. ${ }^{16}$ All measurements were performed by two independent authors using the ruler functionality on IMPAX 6.0 (Agfa). The average of the two values was used in the analysis. PFF type was classified as subcapital, transcervical, basicervical, intertrochanteric or subtrochanteric (Figure 1).

Hierarchical multiple regression (HMR) analysis was performed to allow for stepwise analysis and adjustment for covariates (SPSS v24, IBM). The first model of the HMR analysis contains vitamin D as the dependent variable and medication group as the independent variable. In the second model, sociodemographic factors (age, gender, socioeconomic status and type of residence) were added as independent variables. Fracture type and femoral CCT were added as independent variables in the third model. The $\mathrm{R}^{2}$ change values from the second and third model indicate the degree of correlation between vitamin $\mathrm{D}$ and the independent variables whilst controlling for covariates from previous models. One-way ANOVA was employed to detect seasonal variation in vitamin D levels. Pearson's chi-square was used to compare differences in supplement usage between vitamin D deficient and non-deficient patients, and between institutionalised and non-institutionalised patients. Statistical significance was denoted by a p-value of $<0.05$.

This study has been approved by the Gold Coast Hospital and Health Service Human Research Ethics Committee (Ref: LNR/2019/QGC/48850).

\section{Results:}

Over the 12-month study period, 328 patients presented with a PFF and, of those, 313 patients $(67 \%$ female, $33 \%$ male) received routine vitamin $\mathrm{D}$ testing and were included in the study. The mean age 
of the population was 79.5 years $(\mathrm{SD}=11.0)$, ranging from 43 to 102 years. Vitamin D levels ranged from 9 to $167 \mathrm{nmol} / \mathrm{L}$, with a mean of $61.7 \mathrm{nmol} / \mathrm{L}(\mathrm{SD}=27.5)$. Approximately two-thirds of patients $(\mathrm{n}=208,67 \%)$ had adequate levels of vitamin $\mathrm{D}(\geq 50 \mathrm{nmol} / \mathrm{L})$ and one-third were deficient in vitamin $\mathrm{D}(\mathrm{n}=105,34 \%)$. Due to discrepancies in definitions of vitamin $\mathrm{D}$ deficiency, Figure 2 shows the distribution of serum vitamin $\mathrm{D}$ levels in this cohort to allow for independent interpretation. Furthermore, $116(37 \%)$ patients were in a nursing home or assisted living and 191 (61\%) patients were living independently in the community before admission.

We compared the percentage of patients with vitamin D deficiency in this study with previous reports $^{5-14}$ (Table 1). For each study that we compared to, we adjusted the threshold for vitamin D deficiency in our sample population to match the threshold used in the study. We also adjusted for inclusion criteria, such as age above 65 years, to allow for a meaningful comparison. Our cohort had lower levels of vitamin D deficiency compared to all listed studies.

The three stages of the HMR analysis are summarised in Table 2. In model one, medications accounted for $11 \%$ of the variance in vitamin $D$ levels, which was statistically significant $\left(R^{2}=0.107\right.$, $\mathrm{p}<0.001)$. The mean vitamin $\mathrm{D}$ level was $74.1 \mathrm{nmol} / \mathrm{L}(\mathrm{n}=74)$ in patients receiving calcium and vitamin D supplements only, $73.6 \mathrm{nmol} / \mathrm{L}(\mathrm{n}=42)$ in patients receiving antiresorptive medications, and $54.5 \mathrm{nmol} / \mathrm{L}(\mathrm{n}=197)$ in patients not receiving any bone protection medication. Vitamin $\mathrm{D}$ supplementation, antiresorptive medications or both, were used by $52 \%$ of patients from nursing homes (including assisted living) compared to only $29 \%$ of patients living independently $\left(\chi^{2}=18.52\right.$, $\mathrm{p}=0.001$ ). Out of the 105 patients with vitamin D deficiency and 208 patients with adequate vitamin D levels, 88 (84\%) and 109 (52\%) were not taking vitamin D supplements before admission, respectively. In other words, $15 \%$ of patients taking vitamin D supplements and $45 \%$ of patients not taking vitamin D supplements were deficient in vitamin D (Figure 3). The difference between these two groups were statistically significant $\left(\chi^{2}=29.51, \mathrm{p}<0.001\right)$.

In model two of the HMR analysis, age, gender, socioeconomic index and type of residence (nursing home or private residence) were not significantly correlated with vitamin D levels when controlling for medications $\left(\mathrm{R}^{2}\right.$ change $\left.=0.004, \mathrm{p}=0.9\right)$.

Finally, in the third model, fracture type and femoral CCT had no significant correlation with vitamin $\mathrm{D}$ levels when controlling for covariates in models one and two (age, gender, socioeconomic index, type of residence and medications $)\left(R^{2}\right.$ change $\left.=0.004, p=0.5\right)$.

When testing for seasonal variation, ANOVA revealed vitamin D levels had no statistically significant difference between seasons $(\mathrm{F}(3,309)=1.12, \mathrm{p}=0.3)$. The mean vitamin $\mathrm{D}$ level was $62.3 \mathrm{nmol} / \mathrm{L}$ $(\mathrm{SD}=26.6)$ in summer, $62.0 \mathrm{nmol} / \mathrm{L}(\mathrm{SD}=26.6)$ in autumn, $57.7 \mathrm{nmol} / \mathrm{L}(\mathrm{SD}=26.4)$ in winter, and $65.7 \mathrm{nmol} / \mathrm{L}(\mathrm{SD}=30.5)$ in spring. 


\section{Discussion:}

There are four pertinent findings from these results. Firstly, 34\% of a South-East Queensland cohort of PFF patients were deficient in vitamin D and this is consistently lower than cohorts from other studies conducted around the world. Secondly, sociodemographic factors, fracture type and femoral CCT did not correlate with vitamin D levels in this cohort when accounting for covariates. Thirdly, there was no seasonal variation in vitamin D levels. Lastly, $84 \%$ of vitamin D deficient patients were not taking vitamin D supplements.

South-East Queensland has historically low levels of vitamin D deficiency. In 2011-12, the rate of vitamin D deficiency in the adult Queensland population was the lowest of any state or territory in Australia at $11 \%$, with a nationwide average of $24 \% .{ }^{17}$ The low rates of vitamin D deficiency seen in Queensland are attributed to a combination of factors including the subtropical climate, lifestyle and high UV index. ${ }^{18}$ Consequently, this may explain why there was no statistically significant seasonal variation in vitamin D levels of ageing PFF patients in this study. Inderjeeth and colleagues, ${ }^{14}$ in their Tasmanian study, also reported no statistical seasonal variation and suggested that this may be because PFF patients are older and frailer than the general population and, thus, are less likely to go outdoors regardless of the season.

Living in residential care is a recognised risk factor for vitamin D deficiency. ${ }^{1}$ In a study of 222 PFF patients in Switzerland, Bischoff-Ferrari and co-workers ${ }^{6}$ reported that patients admitted from nursing homes or assisted living had over $20 \mathrm{nmol} / \mathrm{L}$ lower vitamin D levels compared to those admitted from private residences $(p<0.05)$. In our study, admission from a nursing home was not associated with lower levels of vitamin D. This discrepancy may be because the lower sun exposure experienced by nursing home residents was offset by both the higher rates of medication in nursing homes and the higher UV index in Queensland.

Socioeconomic status in this study was not significantly correlated with vitamin D levels after correcting for supplementation. Vitamin D is not substantially different across socially advantaged and disadvantaged areas in the general Australian population, with the proportion of vitamin D deficiency in the first quintile of social disadvantage (26\%) being similar to the fifth quintile (24\%). ${ }^{17}$ However, studies from other countries have found that social deprivation is associated with higher levels of vitamin D deficiency. ${ }^{19,20}$ Several reasons for this have been proposed including reduced physical activity, limited access to supplements and poorer diets in disadvantaged communities. ${ }^{21}$ The discrepancy found in Australia indicates that exposure to sunlight is similar in all patients, irrespective of their socioeconomic status. 
We found no association between vitamin D and femoral cortical thickness. Since it is accepted that cortical thickness corresponds to bone strength and resistance to fracture, ${ }^{22}$ vitamin D levels may not be a reliable indicator for an individual's risk of sustaining a hip fracture. Zhao et al. performed a systematic review and meta-analysis of 33 randomised controlled trials and found that vitamin D supplementation did not reduce fracture incidence in community-dwelling adults. ${ }^{23}$ In contrast, in a nursing home population, a randomised controlled trial found that vitamin $\mathrm{D}$ and calcium supplementation reduced the risk of hip fracture. ${ }^{24,25}$ Our study had a majority of community-dwelling individuals and, thus, the findings reflect the Zhao et al. study.

According to model one of the HMR analysis, calcium and vitamin D supplements were associated with significantly higher levels of serum vitamin D compared to patients not receiving any bone protection medication. Vitamin D supplementation is beneficial in patients with hypovitaminosis D by preventing osteomalacia and reducing the risk of hypocalcemia in patients treated with bisphosphonates. ${ }^{26}$ Furthermore, a retrospective study suggested that bisphosphonates were more effective in raising bone mineral density in patients with vitamin $\mathrm{D}>70 \mathrm{nmol} / \mathrm{L}$ than in patients with lower vitamin D levels. ${ }^{27}$ One-third of this Queensland cohort was deficient in vitamin D and $84 \%$ of these patients were not taking vitamin D supplements. Therefore, while it is unclear how vitamin D may influence fracture risk in this cohort, there are several benefits of supplementation for ageing PFF patients.

There are some limitations to this study. This is a retrospective cohort of patients and there is no healthy control group without fractures. Bone mineral density scans were not routinely obtained and, thus, cortical thickness was used as a surrogate. Additionally, we were limited by the data from patient records and could not account for factors such as comorbidities or medication compliance.

In summary, levels of hypovitaminosis D in ageing PFF patients from South-East Queensland are lower than in other countries and states. Social and demographic factors do not appear to influence vitamin D levels in this cohort. Hypovitaminosis D may not reliably predict an individual's risk of sustaining a low-energy proximal femur fracture. However, routine vitamin D supplementation for ageing PFF patients is beneficial.

\section{IMPACT STATEMENT}

Low levels of vitamin D are not associated with an increased risk of low-energy proximal femur fractures. However, our findings suggest that ageing patients will still benefit from routine vitamin D supplementation irrespective of their social or demographic factors. 


\section{REFERENCES}

1. Nowson CA, McGrath JJ, Ebeling PR, et al. Vitamin D and health in adults in Australia and New Zealand: a position statement. Med J Aust. 2012;196(11):686-687.

https://doi.org/10.5694/mja11.10301.

2. Hoikka V, Alhava EM, Savolainen K, Parviainen M. Osteomalacia in fractures of the proximal femur. Acta Orthop Scand. 1982;53(2):255-260. https://doi.org/10.3109/17453678208992212.

3. Australian Institute of Health and Welfare [webpage]. Hip fracture incidence and hospitalisations in Australia 2015-16. Cat. no. PHE 226. Australian Institute of Health and Welfare. 2018.

https://www.aihw.gov.au/reports/injury/hip-fracture-incidence-in-australia-2015-16. Accessed August $12,2019$.

4. Diamantopoulos AP, Rohde G, Johnsrud I, Skoie IM, Hochberg M, Haugeberg G. The epidemiology of low- and high-energy distal radius fracture in middle-aged and elderly men and women in Southern Norway. PloS one. 2012;7(8):e43367.

https://doi.org/10.1371/journal.pone.0043367.

5. Bakhtiyarova S, Lesnyak O, Kyznesova N, Blankenstein MA, Lips P. Vitamin D status among patients with hip fracture and elderly control subjects in Yekaterinburg, Russia. Osteoporos Int. 2006;17(3):441-446. https://doi.org/10.1007/s00198-005-0006-9.

6. Bischoff-Ferrari HA, Can U, Staehelin HB, et al. Severe vitamin D deficiency in Swiss hip fracture patients. Bone. 2008;42(3):597-602. https://doi.org/10.1016/j.bone.2007.10.026.

7. Sahota O, Gaynor K, Harwood RH, Hosking DJ. Hypovitaminosis D and 'functional hypoparathyroidism' - The NoNoF (Nottingham Neck of Femur) study. Age ageing. 2001;30(6):467472. https://doi.org/10.1093/ageing/30.6.467.

8. Gallacher SJ, McQuillian C, Harkness M, Finlay F, Gallagher AP, Dixon T. Prevalence of vitamin D inadequacy in Scottish adults with non-vertebral fragility fractures. Curr Med Res Opin.

2005;21(9):1355-1361. https://doi.org/10.1185/030079905X59148.

9. Moniz C, Dew T, Dixon T. Prevalence of vitamin D inadequacy in osteoporotic hip fracture patients in London. Curr Med Res Opin. 2005;21(12):1891-1894.

https://doi.org/10.1185/030079905X75023.

10. Bryson DJ, Nichols JS, Ford AJ, Williams SC. The incidence of Vitamin D deficiency amongst patients with a femoral neck fracture: Are current bone protection guidelines sufficient? Acta Orthop Belg. 2013;79(4):470-473.

11. Dhanwal DK, Sahoo S, Gautam VK, Saha R. Hip fracture patients in India have vitamin D deficiency and secondary hyperparathyroidism. Osteoporos Int. 2013;24(2):553-557. https://doi.org/10.1007/s00198-012-1993-y. 
12. Nurmi I, Kaukonen J-P, Lüthje P, et al. Half of the patients with an acute hip fracture suffer from hypovitaminosis D: a prospective study in southeastern Finland. Osteoporos Int. 2005;16(12):20182024. https://doi.org/10.1007/s00198-005-1987-0.

13. Larrosa M, Gomez A, Casado E, et al. Hypovitaminosis D as a risk factor of hip fracture severity. Osteoporos Int. 2012;23(2):607-614. https://doi.org/10.1007/s00198-011-1588-z.

14. Inderjeeth CA, Barrett T, Al-Lahham Y, Mulford J, Nicklason F, Reberger C. Seasonal variation, hip fracture and vitamin D levels in Southern Tasmania. N Z Med J. 2002;115(1152):183-185. 15. Australian Bureau of Statistics. Census of Population and Housing: Socio-Economic Indexes for Areas (SEIFA), Australia, 2016 [webpage]. Cat. no. 2033.0.55.001. Commonwealth of Australia. 2018. https://www.abs.gov.au/AUSSTATS/abs@.nsf/DetailsPage/2033.0.55.0012016. Accessed August 5, 2019.

16. Kumar DA, Anburajan M. The role of hip and chest radiographs in osteoporotic evaluation among south Indian women population: a comparative scenario with DXA. $J$ Endocrinol Invest. 2014;37(5):429-440. https://doi.org/10.1007/s40618-014-0074-9.

17. Australian Bureau of Statistics. Australian Health Survey: Biomedical Results for Nutrients, 201112 [webpage]. Cat. no. 4364.0.55.006. Commonwealth of Australia. 2013. https://www.abs.gov.au/AUSSTATS/abs@.nsf/DetailsPage/4364.0.55.0062011-12. Accessed August $5,2019$.

18. Bureau of Meteorology. Climate Data Online [webpage]. Commonwealth of Australia. 2013. http://www.bom.gov.au/climate/data/index.shtml. Accessed August 2, 2019.

19. Hayden KE, Sandle LN, Berry JL. Ethnicity and social deprivation contribute to vitamin D deficiency in an urban UK population. J Steroid Biochem Mol Biol. 2015;148:253-255. https://doi.org/10.1016/j.jsbmb.2014.11.014.

20. Navarro MdC, Saavedra P, Jódar E, Tejada MJGd, Mirallave A, Sosa M. Osteoporosis and metabolic syndrome according to socio-economic status, contribution of PTH, vitamin D and body weight: The Canarian Osteoporosis Poverty Study (COPS). Clin Endocrinol (Oxf). 2013;78(5):681686. https://doi.org/10.1111/cen.12051.

21. Davis SV. The Relationship Between Socioeconomic Status and Body Mass Index on Vitamin D Levels in African American Women with and without Diabetes Living in Areas with Abundant Sunshine [dissertation]. Graduate Theses and Dissertations. 2013. https://scholarcommons.usf.edu/etd/4660. Accessed August 14, 2019. 22. Augat $\mathrm{P}$, Schorlemmer S. The role of cortical bone and its microstructure in bone strength. Age Ageing. 2006;35 Suppl 2:ii27-ii31. https://doi.org/10.1093/ageing/afl081.

23. Zhao JG, Zeng XT, Wang J, Liu L. Association Between Calcium or Vitamin D Supplementation and Fracture Incidence in Community-Dwelling Older Adults: A Systematic Review and Metaanalysis. JAMA. 2017;318(24):2466-2482. https://doi.org/10.1001/jama.2017.19344. 
24. Chapuy MC, Arlot ME, Duboeuf F, et al. Vitamin D3 and Calcium to Prevent Hip Fractures in Elderly Women. N Engl J Med. 1992;327(23):1637-1642.

https://doi.org/10.1056/nejm199212033272305.

25. Chapuy MC, Pamphile R, Paris E, et al. Combined calcium and vitamin D3 supplementation in elderly women: confirmation of reversal of secondary hyperparathyroidism and hip fracture risk: the Decalyos II study. Osteoporos Int. 2002;13(3):257-264. https://doi.org/10.1007/s001980200023.

26. Rosen CJ, Brown S. Severe Hypocalcemia after Intravenous Bisphosphonate Therapy in Occult Vitamin D Deficiency. N Engl J Med. 2003;348(15):1503-1504.

https://doi.org/10.1056/NEJM200304103481521.

27. Deane A, Constancio L, Fogelman I, Hampson G. The impact of vitamin D status on changes in bone mineral density during treatment with bisphosphonates and after discontinuation following longterm use in post-menopausal osteoporosis. BMC Musculoskelet Disord. 2007;8(1):3.

https://doi.org/10.1186/1471-2474-8-3. 


\section{TABLES}

Table 1: Comparison to other studies investigating vitamin D levels in proximal femur fracture patients

\begin{tabular}{|c|c|c|c|c|}
\hline Study & Location & $\begin{array}{l}\text { Vitamin D } \\
\text { deficiency cut- } \\
\text { off value } \\
(\text { nmol/L) }\end{array}$ & $\begin{array}{l}\text { Proportion of } \\
\text { subjects with } \\
\text { vitamin D } \\
\text { deficiency (\%) }\end{array}$ & $\begin{array}{l}\text { Proportion of } \\
\text { matched subjects } \\
\text { with vitamin D } \\
\text { deficiency in our } \\
\text { cohort }(\%)\end{array}$ \\
\hline Bakhtiyarova et al. $^{5}$ & Russia & $<25$ & 65 & 9 \\
\hline $\begin{array}{l}\text { Bischoff-Ferrari et } \\
\text { al. }{ }^{6}\end{array}$ & Switzerland & $<50$ & 80 & 31 \\
\hline Bryson et al. ${ }^{10}$ & $\begin{array}{l}\text { Leicester, United } \\
\text { Kingdom }\end{array}$ & $<30$ & 71 & 13 \\
\hline Dhanwal et al. ${ }^{11}$ & India & $<50$ & 77 & 33 \\
\hline Gallacher $^{8}$ & Scotland & $<50$ & 92 & 32 \\
\hline Inderjeeth et al. ${ }^{14}$ & Tasmania & $<28$ & 68 & 11 \\
\hline Larrosa et al. ${ }^{13}$ & Spain & $<62$ & 67 & 51 \\
\hline Moniz et al. ${ }^{9}$ & London & $<50$ & 82 & 33 \\
\hline Nurmi et al. ${ }^{12}$ & Finland & $<37.5$ & 53 & 20 \\
\hline Sahota et al. ${ }^{7}$ & $\begin{array}{l}\text { Nottingham, United } \\
\text { Kingdom }\end{array}$ & $<30$ & 68 & 12 \\
\hline
\end{tabular}


Table 2: Summary of hierarchical multiple regression analysis between vitamin $D$ and medications, age, sex, season of admission, socioeconomic status, nursing home status, fracture type and femoral CCT

\begin{tabular}{|c|c|c|c|c|c|c|}
\hline \multirow[t]{2}{*}{ Variable } & \multicolumn{2}{|l|}{ Model 1} & \multicolumn{2}{|l|}{ Model 2} & \multicolumn{2}{|l|}{ Model 3} \\
\hline & $\beta(95 \%$ CI) & p-value & $\beta(95 \%$ CI) & p-value & $\beta(95 \%$ CI $)$ & p-value \\
\hline Medications & $-10.7(-14.1,-7.2)$ & $<0.001$ & $-10.2(-13.8,-6.6)$ & $<0.001$ & $-10.1(-13.8,-6.5)$ & $<0.001$ \\
\hline Age & & & $0.0(-0.3,0.3)$ & 0.9 & $0.1(-0.3,0.4)$ & 0.7 \\
\hline Sex & & & $2.5(-3.9,8.8)$ & 0.4 & $3.1(-3.3,9.6)$ & 0.3 \\
\hline $\begin{array}{l}\text { Season of } \\
\text { admission }\end{array}$ & & & $0.5(-2.1,3.2)$ & 0.7 & $0.6(-2.1,3.3)$ & 0.7 \\
\hline $\begin{array}{l}\text { Socioeconomic } \\
\text { status }\end{array}$ & & & $0.0(-0.1,0.2)$ & 0.7 & $0.03(-0.2,0.2)$ & 0.8 \\
\hline $\begin{array}{l}\text { Nursing home } \\
\text { status }\end{array}$ & & & $2.3(-4.4,9.0)$ & 0.5 & $2.30(-4.4,9.0)$ & 0.5 \\
\hline Fracture type & & & & & $0.0(-2.2,2.2)$ & 0.9 \\
\hline Femoral CCT & & & & & $0.3(-0.2,0.6)$ & 0.2 \\
\hline $\mathbf{R}^{2}$ & 0.107 & & 0.111 & & 0.115 & \\
\hline $\mathbf{R}^{2}$ change & 0.107 & & 0.004 & & 0.004 & \\
\hline P-value & $<0.001$ & & 0.9 & & 0.5 & \\
\hline
\end{tabular}




\section{FIGURE LEGENDS:}

Figure 1 - Classification of proximal femur fractures

Subcapital fractures occur at the junction of the head and neck of femur. Transcervical fractures occur in the middle of the neck of femur. Basicervical fractures occur at the base of the neck of femur.

Intertrochanteric fractures involve the greater and/or lesser trochanter. Subtrochanteric fractures occur in the femoral shaft up to $5 \mathrm{~cm}$ distal to the lesser trochanter.

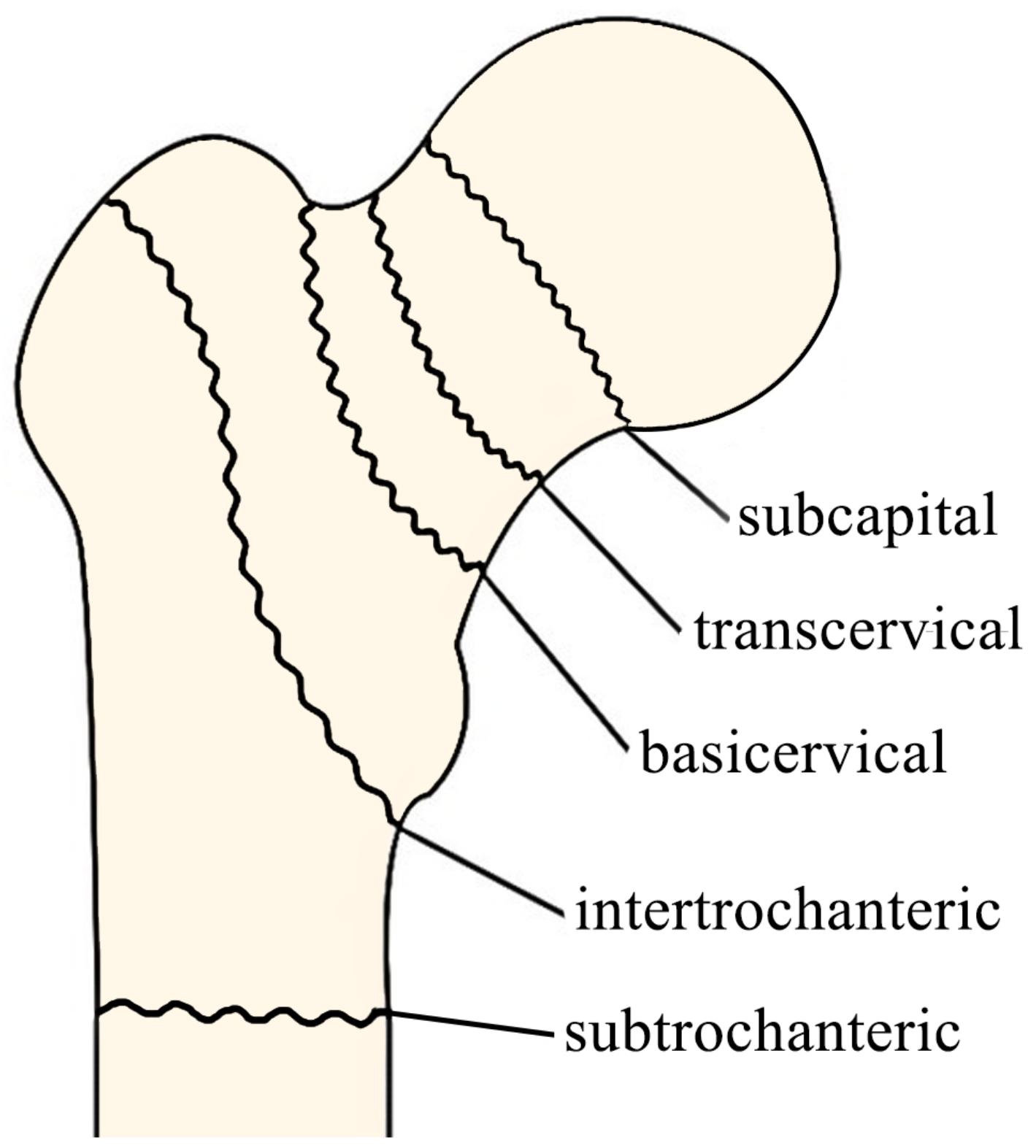


Figure 2 - Distribution of serum vitamin D levels

Distribution of serum vitamin D levels in the studied cohort.

Figure 2: Distribution of serum vitamin D levels

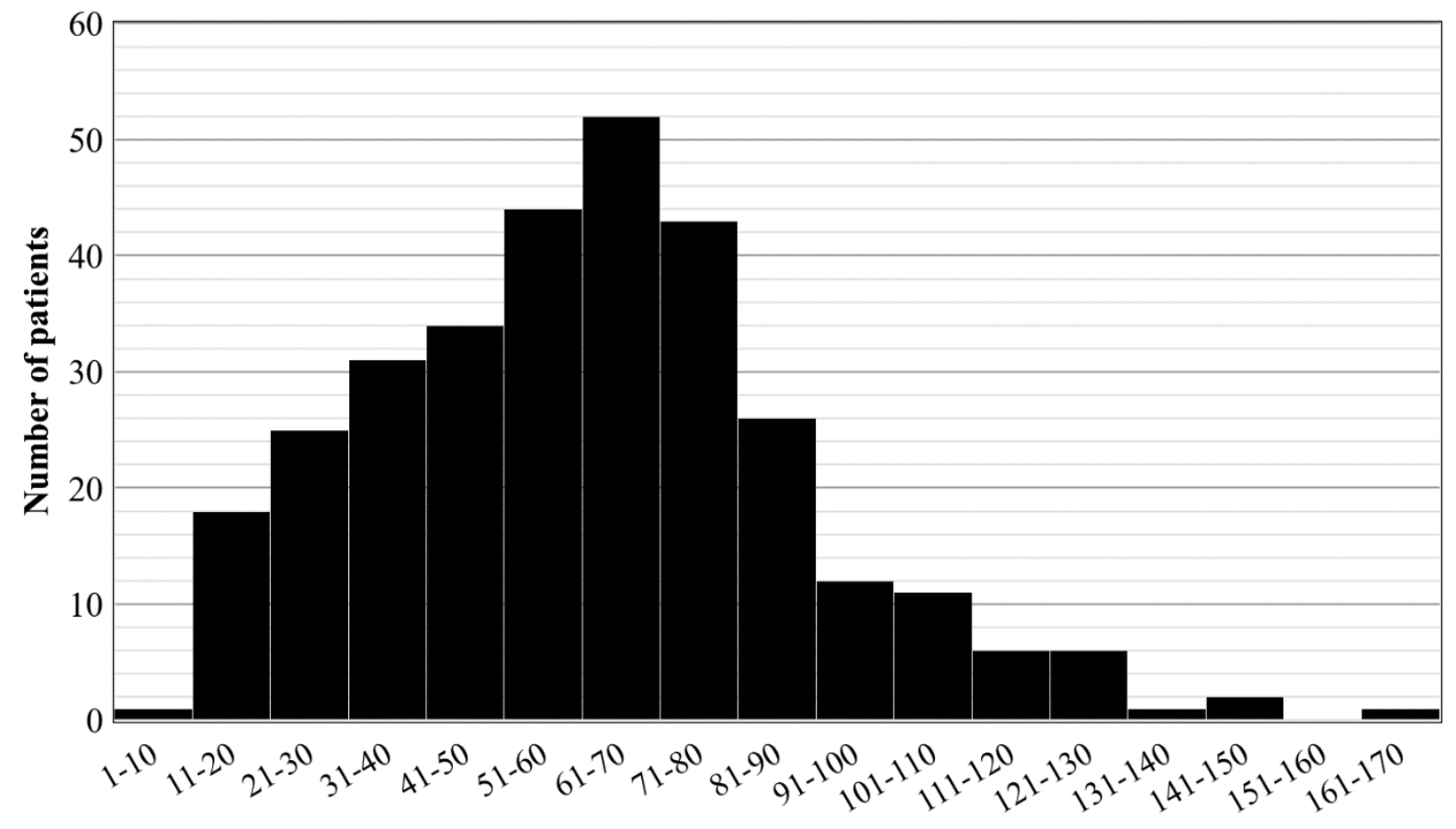

Serum vitamin D level (nmol/L) 
Figure 3 - Proportion of patients with vitamin D deficiency by supplement usage

The number of patients with a vitamin D deficiency $(<50 \mathrm{nmol} / \mathrm{L})$ or adequate vitamin $\mathrm{D}(\geq 50 \mathrm{nmol} / \mathrm{L})$ grouped by vitamin D supplement usage. The differences between the two groups were statistically significant $\left(\chi^{2}=29.51, \mathrm{p}<0.001\right)$.

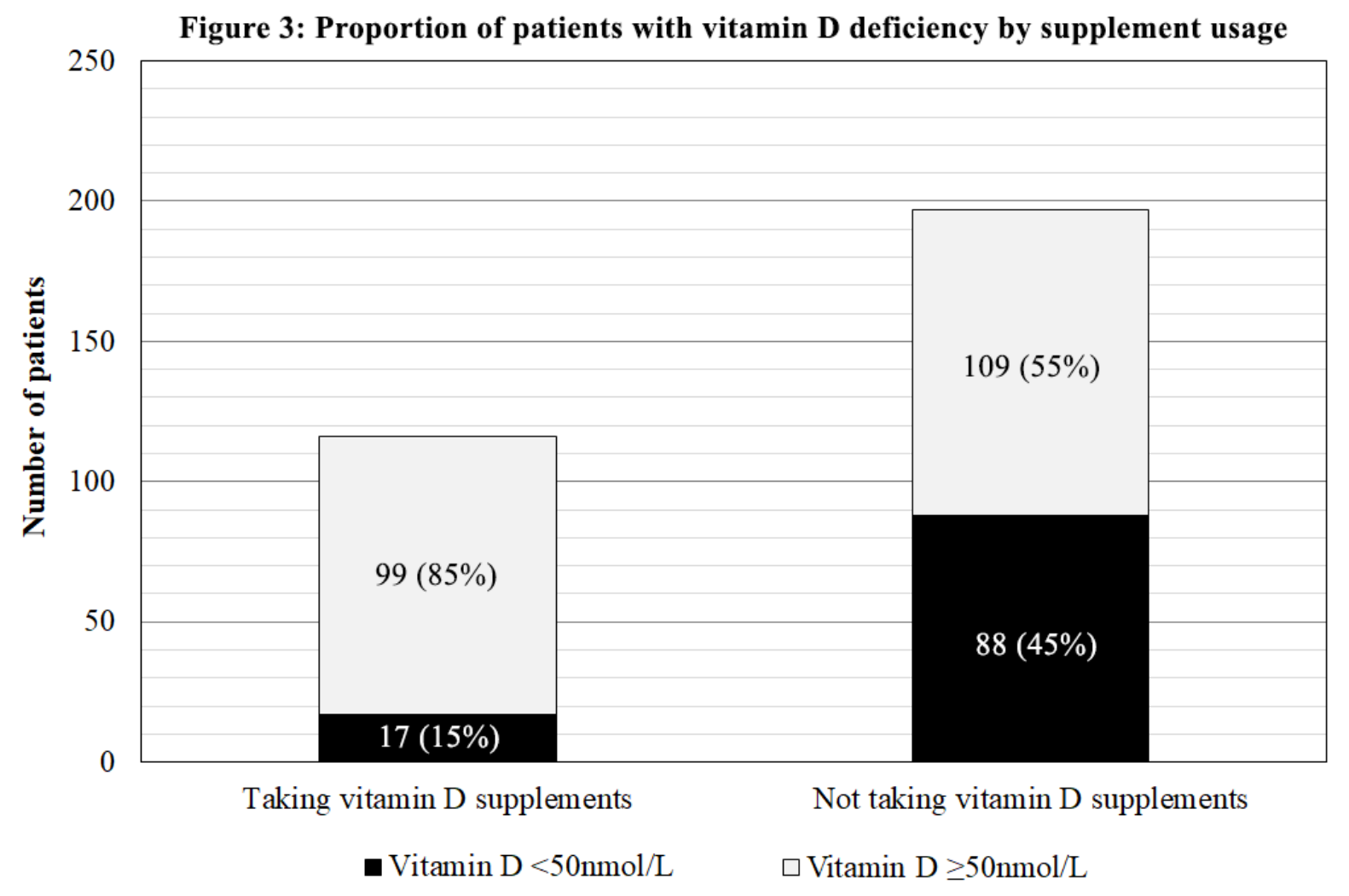

